\title{
The Effect of Radical Trap Moieties on Electron Capture Dissociation Spectra of Substance $\mathbf{P}$
}

\author{
Marina A. Belyayev and Jason J. Cournoyer \\ Department of Chemistry, Boston University, Boston, Massachusetts, USA
}

\author{
Cheng Lin and Peter B. O'Connor \\ Mass Spectrometry Resource, Department of Biochemistry, Boston University School of Medicine, Boston, \\ Massachusetts, USA
}

\begin{abstract}
To further test the hypothesis that electron capture dissociation (ECD) involves long-lived radical intermediates and radical migration occurs within these intermediates before fragmentation, radical trap moieties were attached to peptides with the assumption that they would reduce fragmentation by decreasing the mobility of the radical. Coumarin labels were chosen for the radical traps, and unlabeled, singly-labeled, and doubly-labeled Substance P were analyzed by ECD. The results demonstrated a correlation between the number and position of tags on the peptide and the intensity of side-chain cleavages observed, as well as an inverse correlation between the number of tags on the peptide and the intensity of backbone cleavages. Addition of radical traps to the peptide inhibits backbone cleavages, suggesting that either radical mobility is required for these cleavages, or new noncovalent interactions prevent separation of backbone cleavage fragments. The enhancement of side-chain cleavages and the observation of new side-chain cleavages associated with aromatic groups suggest that the gas-phase conformation of this peptide is substantially distorted from untagged Substance P and involves previously unobserved interactions between the coumarin tags and the phenylalanine residues. Furthermore, the use of a double resonance (DR)-ECD experiment showed that these side-chain losses are all products of long-lived radical intermediate species, which suggests that steric hindrance prevents the coumarin-localized radical from interacting with the backbone while simultaneously increasing the radical rearrangements with the side chains. (J Am Soc Mass Spectrom 2006, 17, 1428-1436) (c) 2006 American Society for Mass Spectrometry
\end{abstract}

$\mathrm{E}$ lectron capture dissociation (ECD), a relatively new tandem mass spectrometry fragmentation technique, plays a useful role in protein sequencing and identification/localization of posttranslational modifications due to the backbonedirected nature of the cleavages generated. ECD targets $\mathrm{N}-\mathrm{C}_{\alpha}$ bonds [1, 2] and disulfide bonds, [3], but preserves post-translational modifications [4] such as phosphorylation $[5,6]$ and glycosylation $[7$, 8]. ECD is capable of discriminating between conformers and, thus, allows for direct observation and analysis of intermediate and unfolded states [9-11]. ECD also provides complementary data to collision activated dissociation (CAD) [3].

ECD involves reactions between multiply charged peptide ions and low-energy electrons [1, 3], which result in rapid backbone fragmentation and in the

Published online July 27, 2006

Address reprint requests to Professor P. B. O'Connor, Mass Spectrometry Resource, Department of Biochemistry, Boston University School of Medicine, 715 Albany St., R806, Boston, MA 02118, USA. E-mail: poconnor@ bu.edu creation of radicals that further propagate intramolecularly and induce numerous cleavages throughout the peptide [12]. Generally, ECD results in three types of cleavages: backbone, side-chain groups, and small molecule losses. Although backbone cleavages $\mathrm{N}$-terminus to proline have never been reported [13], one of the most important characteristics of ECD is its relative non-specificity [3]. In spite of its clear utility, the mechanism of ECD is still under vigorous debate $[2,9,12$, 14-18].

Currently, it is believed that the primary mechanism of ECD involves nonergodic backbone cleavage at $\mathrm{N}-\mathrm{C}_{\alpha}$ bonds to produce $\mathrm{c} / \mathrm{z}$ ions. Alternatively, Syrstad et al. proposed the formation of a superbase, which then abstracts a sterically proximate proton from a hypervalent amide group as the primary fragmentation mechanism $[14,16,19]$, and they were able to develop full reaction coordinate energetic diagrams with transition-state barriers and the thermodynamic energies in a small system using ab initio calculations, which showed that the radical intermediate was stabilized by the presence of nearby positive charges. However, $\mathrm{H} \cdot$ transfer, whether direct or 


\section{(a)}

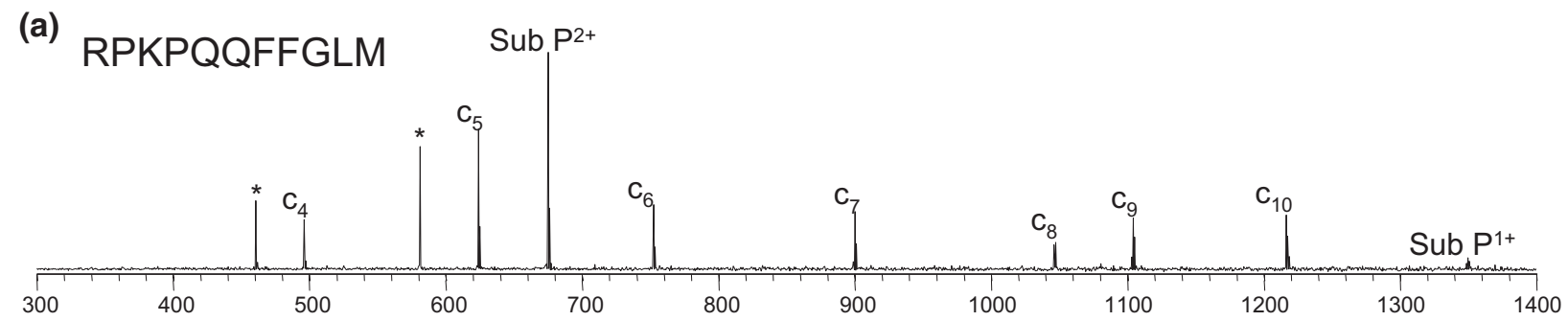

\section{(b) RPK $^{\mathrm{t}} \mathrm{PQQFFGLM}$}

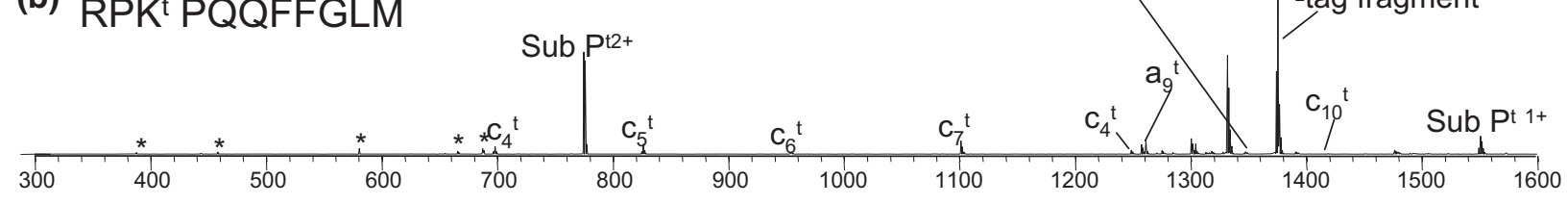

\section{(c) ${ }^{\text {RPPKPQQFFGLM }}$}

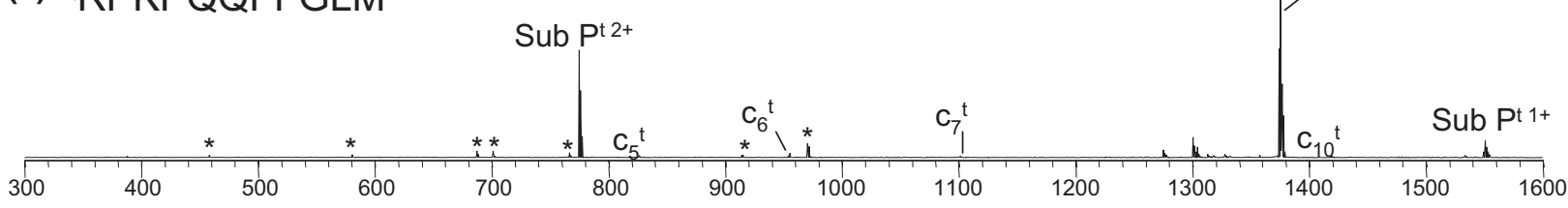

(d) ${ }^{t}$ RPKt PQQFFGLM

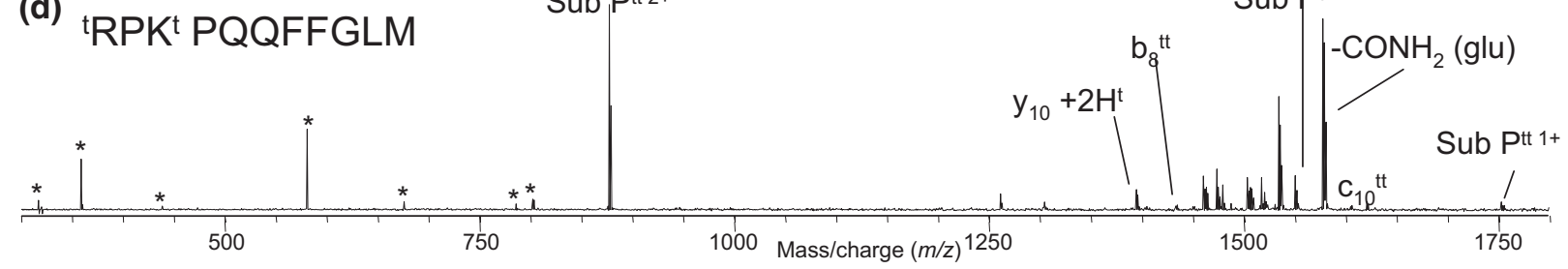

Figure 1. (a) ECD spectrum of Substance P; (b) ECD spectrum of doubly-Coumarinated Substance P; (c) ECD spectrum of singly-Coumarinated Substance P on the lysine side chain; (d) ECD spectrum of singly-Coumarinated Substance P on the $\mathrm{N}$-terminus. An asterisk indicates electronic noise or harmonics in the spectra.

via a superbase, does not account for all observed ECD fragments. A secondary mechanism that involves radical propagation along the peptide backbone via a cascade reaction was proposed to explain secondary backbone fragments that were observed with cyclic peptides [12]. Secondary fragmentation pathways have recently been shown to take place over a range of time scales [20]. For example, cyclosporine-A secondary fragments formed faster than $100 \mu \mathrm{s}$ but some gramicidin-S secondary fragments formed after $1 \mathrm{~ms}$ [20].

The radical cascade mechanism requires a mobile radical to be present in a long-lived radical intermediate, which is supported by H/D scrambling observations [17]. To further test this mechanism, a coumarin radical trap was synthetically attached to Substance P. If a long-lived radical is involved in the fragmentation, a radical trap should suppress fragmentation. A tag useful for mass spectrometry analysis should label the desired peptide quickly and reproducibly with high reaction yields and minimal side reactions, and should be stable under analytical conditions for analysis by Fourier transform mass spectrometer (FTMS) and under the conditions necessary for separation and purifi- cation [21]. A coumarin based tag was chosen due to its conjugated structure and simple reaction mechanism with the peptide Substance P utilizing N-hydroxy succinimide (NHS) ester chemistry, which tags the peptide at the $\mathrm{N}$-terminus and the amine group of the lysine side chain. The fragmentation patterns of singly-tagged Substance P were studied for both labeling sites (separated by HPLC). Double resonance (DR)-experiments were used with ECD to determine which fragments were derived from long-lived intermediates and, hence, to suggest possible conformations of the peptide during cleavage.

\section{Methods}

All chemicals were purchased from Sigma (St. Louis, $\mathrm{MO}$ ). The coumarin tag (7-methoxycoumarin-3-carboxylic acid succinimidyl ester, product number M1410, Invitrogen, Carlsbad, CA) was a kind gift from Anna Pashkova and Barry Karger from Northeastern University. A stock solution $\left(10^{-4} \mathrm{M}\right)$ of Substance $\mathrm{P}$ was made from the peptide. To tag the peptide, $2 \mu \mathrm{L}$ of $1 \mathrm{M}$ ammonium acetate was added to $10 \mu \mathrm{L}$ of the $10^{-4} \mathrm{M}$ 


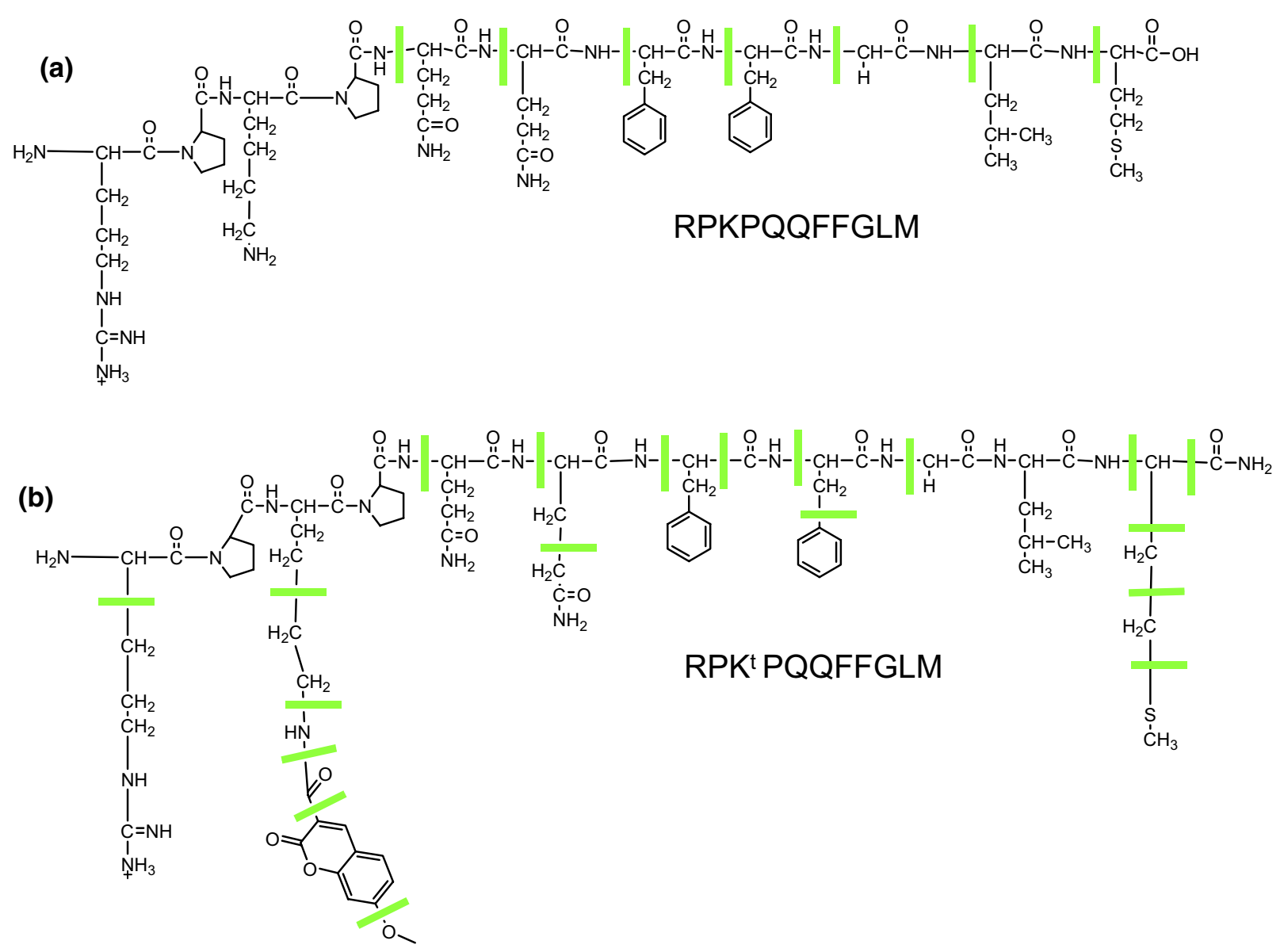

Figure 2. (a) Cleavage patterns of Substance P; (b) Cleavage patterns of doubly-Coumarinated Substance P; (c) Cleavage patterns of singly-Coumarinated Substance P on the lysine side chain; (d) Cleavage patterns of singly-Coumarinated Substance P on the $\mathrm{N}$-terminus.

peptide solution. Meanwhile, $10 \mu \mathrm{g}$ of solid coumarin tag was dissolved in $100 \mu \mathrm{L} \mathrm{DMF}$, and $5 \mu \mathrm{L}$ of the freshly prepared tag solution was added to $12 \mu \mathrm{L}$ of the peptide solution. The reaction was carried out with room temperature at constant stirring for $40 \mathrm{~min}$.

All ECD experiments were performed on a homebuilt FTMS described previously [22, 23]. The peptides were electrosprayed in 50:50 $\mathrm{MeOH} /$ water solution with $1 \%$ formic acid at $\sim 10^{-6} \mathrm{M}$, and precursor ions were isolated using the front-end quadrupole (Q1). The ions transmitted into the cell were trapped using gatedtrapping, and were then irradiated with low-energy $(\sim 0.3 \mathrm{eV})$ electrons for 100 to $200 \mathrm{~ms}$. A conventional FTMS excitation/detection sequence averaging 20 scans was used to detect fragment ions. During DR-ECD data acquisition, the charge reduced species were ejected from the cell by applying a single frequency excitation with an amplitude of $40 \mathrm{~V}$ during the electron irradiation period.

Separation of the $\mathrm{N}$-terminus and lysine tagged Substance P was performed by reversed-phase HPLC (solvent module 125 with detector module 166, Beckman Coulter, Fullerton, CA) equipped with a $20 \mu \mathrm{L}$ injection loop and $4.6 \times 250 \mathrm{~mm} \mathrm{C18}$ column (Vydak,
Hesperia, CA). Mobile phase A consisted of 5:95 acetonitrile/water with $0.05 \%$ trifluoroacetic acid (TFA) and mobile phase B consisted of 80:20 acetonitrile/water with $0.035 \%$ TFA. A gradient of 0 to $40 \%$ B in $60 \mathrm{~min}$ was used to separate the two species of tag. Fractions were dried using a Speedvac with refrigerated vapor trap (Savant RVT 4104, Thermo Electron Corp., Milford, MA) several times in water to remove TFA for FTMS analysis.

\section{Results and Discussion}

Figure 1 depicts the ECD spectra of the untagged peptide (Figure 1a), the singly-tagged peptides (Figure $1 \mathrm{~b}$ and $\mathrm{c}$ ), and the doubly-tagged peptide (Figure 1d) (insets: the superscript " $\mathrm{t}$ " indicates the tag position). The cleavages observed in these four spectra are also plotted in Figure 2. Figure 1a shows a typical Substance $P$ ECD spectrum. The spectrum yields seven out of eight possible backbone cleavages; the proline $\mathrm{N}-\mathrm{C}_{\alpha}$ cleavage cannot be observed [13]. These cleavages are primarily c-type ions (attributable to the $\mathrm{N}$-terminal charge), but a tiny peak corresponding to the $z_{9}$ ion (unlabeled) is visible at $\mathrm{m} / \mathrm{z} \sim 1080$. Two isomers of 


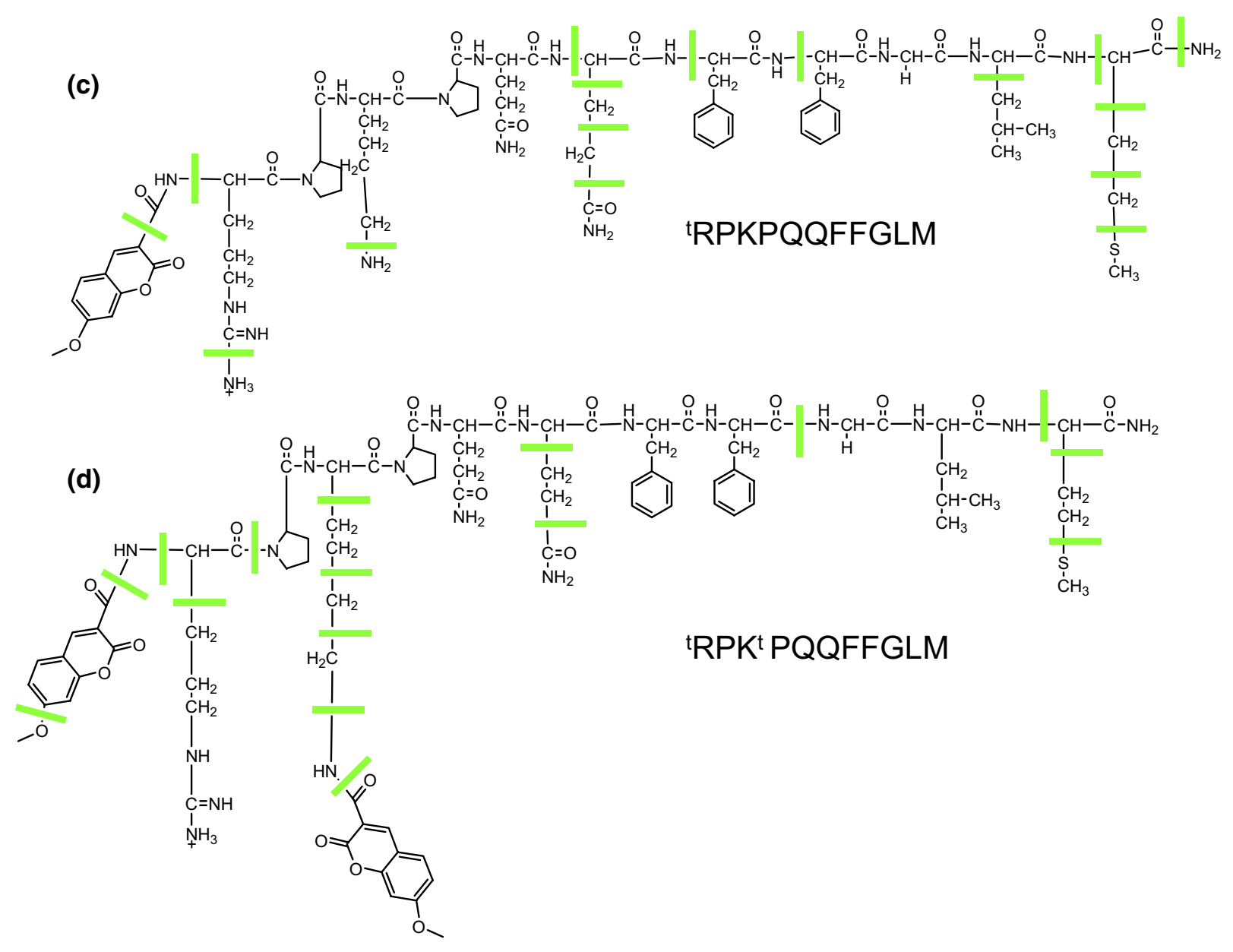

Figure 2. Continued.

singly-tagged peptides are present: lysine-tagged Substance $\mathrm{P}$ (Figure $\mathrm{lb}$ ) and $\mathrm{N}$-terminus tagged Substance $\mathrm{P}$ (Figure 1c). Lysine-tagged Substance P produces six backbone c-type cleavages and one a-type cleavage, but the $\mathrm{N}$-terminus-tagged peptide generates only four ctype ions. In both spectra, these backbone cleavages are reduced in intensity compared with the unlabeled peptide (Figure 1a). Figure 1d shows the ECD spectrum of the doubly tagged Substance P. This spectrum shows very few backbone cleavages at even more diminished intensities, and side-chain cleavages at higher intensities. For Figure 1b, c, and d, the side-chain cleavages usually involve multiple losses of various groups, which are summarized in the plots in Figure 2 and tabulated in Supplementary Material section tables (which can be found in the electronic version of this article). Figure 3 expands the side-chain cleavage regions from Figure 1.

Figure 4 shows the results from a DR-ECD experiment on the tagged Substance $\mathrm{P}$ in which the charge reduced molecular ion $[\mathrm{M}+2 \mathrm{H}]^{+\cdot}$ is continuously ejected from the ICR cell during the ECD experiment [20]. Figure 4a shows the ECD spectrum of the singlytagged Substance P. Figure $4 \mathrm{~b}$ shows the DR-ECD experiment of the singly-tagged species from Figure $4 \mathrm{a}$ with the resonant ejection applied at $m / z 1550$, which is the $\mathrm{m} / \mathrm{z}$ of the charge reduced molecular ion. In this case, the two variants of the singly-tagged species are not separated, so this is the ECD spectrum of the mixture of the N-terminally tagged and lysine tagged species. Figure $4 \mathrm{c}$ shows the ECD spectrum and Figure $4 \mathrm{~d}$ shows the DR-ECD spectrum, of the doubly tagged Substance P. In both DR-ECD experiments, the peaks resulting from side-chain cleavages are dramatically reduced in intensity. In Figure $4 b$, the fragment ions resulting from backbone cleavages $c_{5}$ to $c_{10}$ experience, for the most part, minimal abundance changes, but $c_{4}$ is eliminated from the spectrum. In Figure $4 d$, the sidechain loss peaks around $\mathrm{m} / \mathrm{z} 1500$ are completely eliminated with double resonance.

The addition of the coumarin tag, which contains a large $\mathrm{sp}^{2}$ conjugated system, resulted in dramatic reduction in backbone fragments. The intensities of the backbone cleavage peaks are diminished in spectra of singly-tagged and doubly tagged species. The coumarin tag's delocalized electronic structure serves as a radical trap by providing sites where a radical can be resonance stabilized. The lower intensities of backbone fragment peaks can be attributed to the electron capture in the Rydberg state followed by internal conversion to a low 

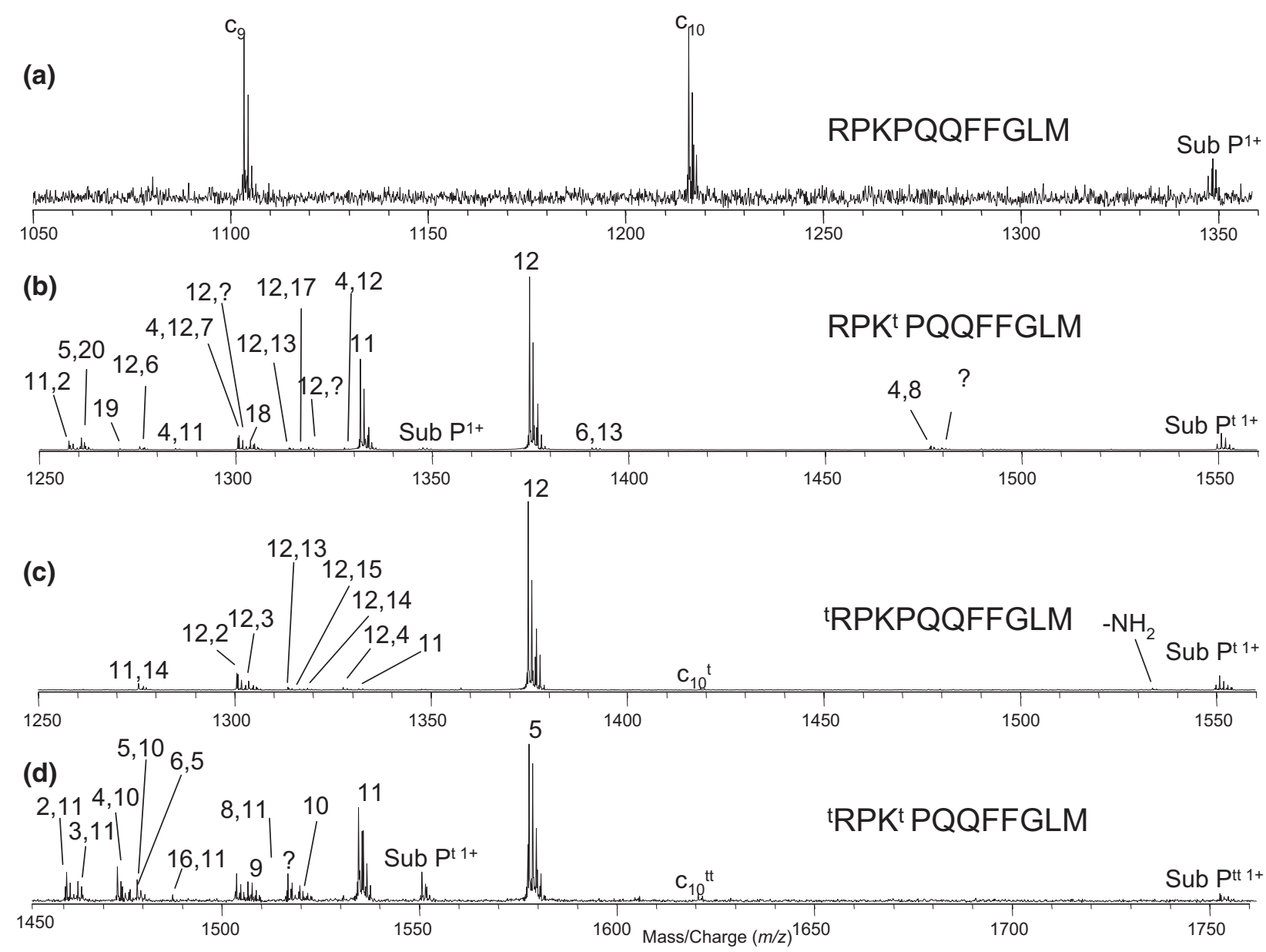

Figure 3. (a) 1050-1360 m/z: Region of expected side-chain cleavages of Substance P; (b) 1450-1760 $\mathrm{m} / \mathrm{z}$ : Region of expected side-chain cleavages of doubly-coumarinated Substance P; (c) 1250-1560 m/z: Region of expected side-chain cleavages of singly-coumarinated Substance P on the lysine side chain; (d) 1250-1560 m/z: Region of expected side-chain cleavages of singly-coumarinated Substance P on the $N$-terminus.

electronic, but vibrationally excited, state. A large conjugated system such as the coumarin tag usually involves more closely-spaced energy levels compared with a $\mathrm{sp}^{3}$ hybridized system. Thus, internal conversion that requires resonant interaction of two states, from the Rydberg state to a low lying electronic state within the coumarin tag is highly probable, which may account for the electron landing in the local energy minimum of the tag rather than at the charge sites. The resonance stabilization of the coumarin tag provides a local potential minimum, which stabilizes the electron and thus retains it at the coumarin site.

In singly-tagged Substance $\mathrm{P}$, with the tag residing either on the $N$-terminus or the side chain of the lysine residue, the side-chain cleavages included fragments from arginine, lysine, glutamine, methionine, leucine, and phenylalanine. In particular, the $\mathrm{C}_{6} \mathrm{H}_{5} \cdot$ neutral loss, previously unreported, was observed from the lysine tagged species. The sidechain fragmentation mechanisms of arginine, lysine, glutamine, and methionine are discussed by Cooper et al. [24] and that of leucine by
Kjeldsen et al. [25]. The proposed mechanism of fragmentation of the phenylalanine side chain is under speculation however, the $\pi$-bonded structure of the tag offers an intriguing possibility. The conjugated systems of the tag and the two phenylalanine side chains have the potential to undergo $\pi$-stacking interactions (and would be expected to do so in solution phase). $\pi$-Stacking of the coumarin moiety and the phenylalanine side chains could enable radical transfer onto the phenylalanine side chain (Figure 5), to result in the loss of a phenyl radical group. However, the low abundance of the fragment resulting from this loss suggests that $\pi$-stacking in the gas phase is not a strong interaction.

Other small molecule losses from both singly-tagged peptides included carbon monoxide and ammonia losses, both of which have been previously reported and mechanisms for their losses discussed [24, 26, 27]. Finally, the singly-tagged peptides contained losses of the tag as well as a tag fragment. The mixture of singly-tagged Substance P was separated via HPLC and although, with ECD, no backbone cleavages were gen- 


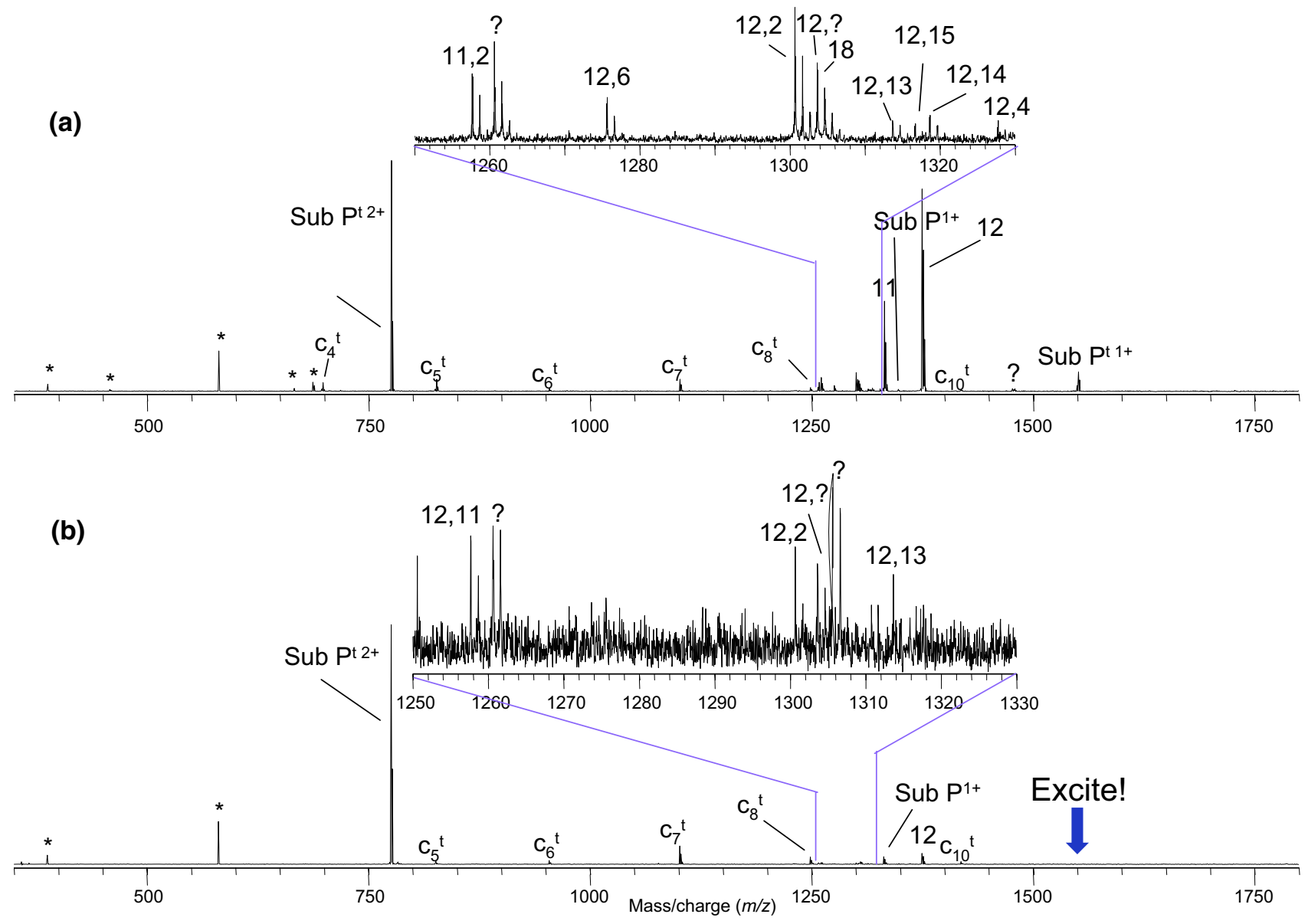

Figure 4. (a) The ECD spectrum of mixed singly-Coumarinated Substance P without ejection; (b) DR-ECD spectrum of mixed singly-Coumarinated Substance P with ejection at $m / z 1550$; (c) The ECD spectrum of doubly-Coumarinated Substance P without ejection; (d) DR-ECD spectrum of doublyCoumarinated Substance P with ejection at $\mathrm{m} / \mathrm{z} 1753 \mathrm{~m} / \mathrm{z}$. An asterisk indicates electronic noise or harmonics in the spectra.

erated that could be used to decipher between the lysine and $N$-terminus tagged peptides, losses of the tag attached to parts of the lysine side chain present in one spectrum and the lack of such losses in the other spectrum served as indicators to distinguish between the two species. CAD was also used to confirm this assignment (data not shown).

The doubly-tagged Substance P, with coumarin groups attached at both the $\mathrm{N}$-terminus and the lysine side chain, produced fragments from the side chains of arginine, glutamine, lysine, and methionine. Small molecule losses from the doubly tagged peptide included loss of $\mathrm{C}-\mathrm{O}$ from a tag as well as the loss of a tag fragment and one or both tags, as previously described.

The significant reduction of backbone cleavages apparent with the sequential addition of radical traps to Substance P strongly correlates with the concept that the radical is involved in secondary cleavages, as previously suggested [12]. It is proposed that the coumarin tag uses its highly conjugated system to act as a radical trap and captures the radical, decreasing the radical's probability of interacting with the backbone carbonyl. Thus, radical trapping results in stabilization of the backbone carbonyls and the backbone cleavages are significantly reduced. At the same time, the conjugated system of the coumarin tag is able to freely rotate on the single bond connecting it to the rest of the peptide. As it bends, the amino acid side-chain groups are sterically more accessible to the radical residing on the tag, creating possible pathways of radical reactions on the side chains of the amino acids and, over time, inducing cleavages in the side chains.

Additional evidence of a radical cascade mechanism can be derived from the results of DR-ECD of native Substance P (not shown) and the singly- and doublylabeled peptides (Figure 4). The singly-labeled Substance P used for DR-ECD consisted of a mixture of lysine and $\mathrm{N}$-terminus tagged Substance P. The spectra of singly-tagged Substance P show that most backbone cleavages were not affected by the resonant ejection, while the side-chain cleavages were substantially reduced or eliminated. Reduction of these fragment ion 


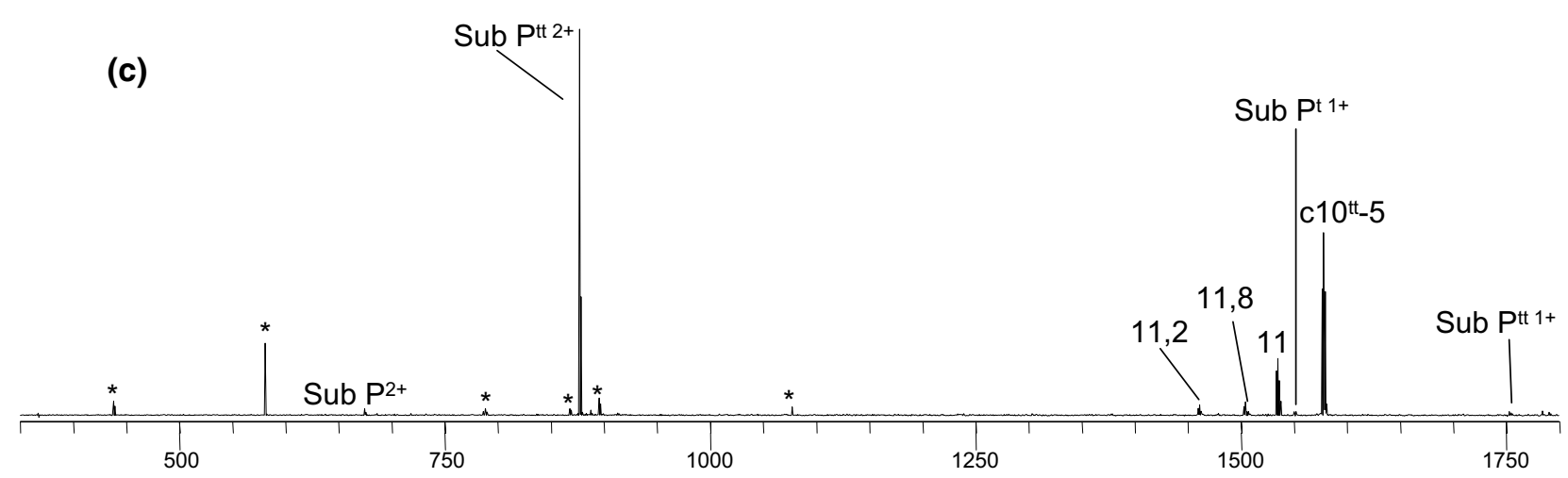

(d)

Sub $\mathrm{P}^{\mathrm{tt} 2+}$

Figure 4. Continued.

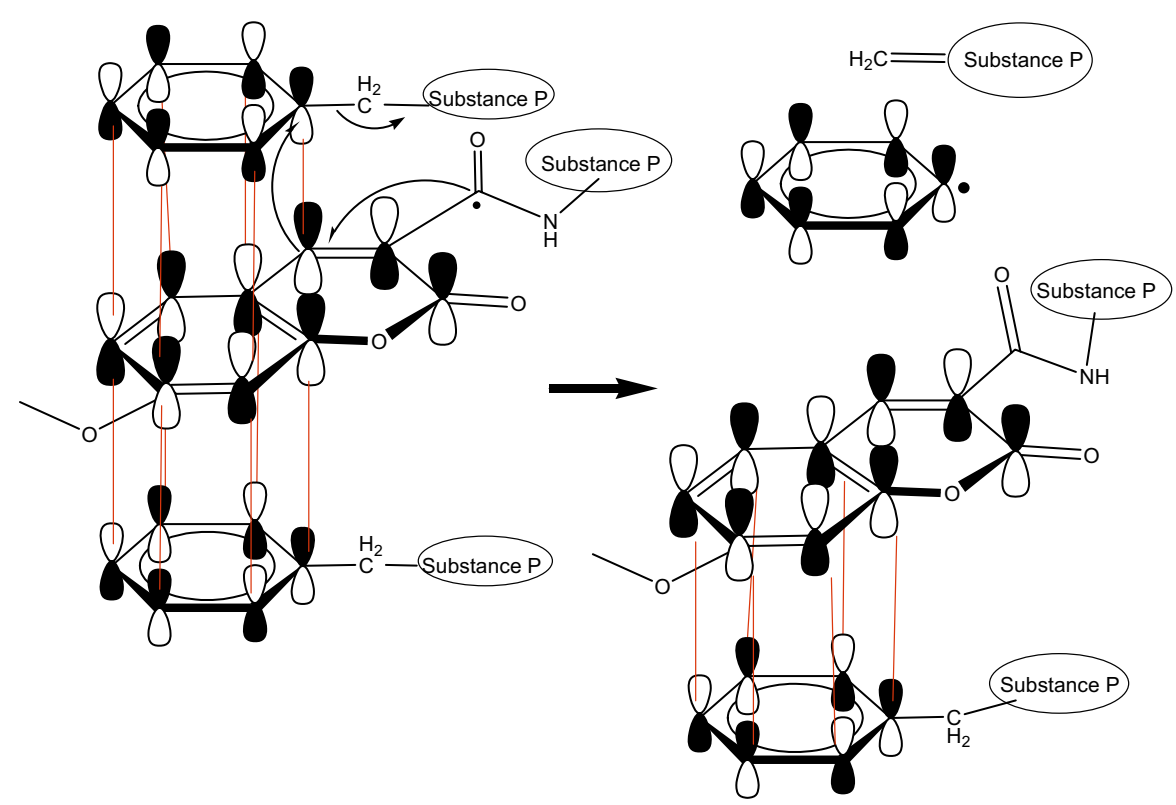

Figure 5. Proposed mechanism for formation of the $\mathrm{C}_{6} \mathrm{H}_{5}$. neutral loss involving $\pi$-stacking of the coumarin tag with the phenylalanine side chains. 
abundances upon resonant ejection of the charge reduced molecular ion indicates that these side-chain cleavages are generated on a time period greater than the time it takes to excite the intermediate to $\sim 1 / 2$ the cell radius-typically on the order of $\sim 100 \mu \mathrm{s}$.

The numerous observed fragments indicate that the secondary fragments observed in ECD involve radical propagation [12]. However, the exact mechanism of cleavage is complicated by the juxtaposition of decreasing intensities of backbone cleavages with increasing intensities of side-chain cleavages (Figure 3). If the secondary mechanism involved only radical propagation, then the amounts of all cleavages would be expected to decrease in the presence of a radical trap. Thus, the increase in abundance of slow side-chain cleavages indicate the presence of another relevant mechanism. The ability of the coumarin tag groups to participate in $\pi$-stacking suggests that the flexibility of the tag is important, enabling the radical to interact with amino acid side chains and causing radical induced cleavage at these locations. Hence, the most likely mechanism of ECD involves two stages: after initial electron capture in a high Rydberg state, the electron, as it slowly drops down the potential well onto the peptide, undergoes internal conversion to a local potential minimum, which is a vibrationally excited, low-lying electronic state. When this local minimum is on the carbonyl, as in the unmodified Substance P, the traditional rapid ECD c/z-type cleavages are generated via $\alpha$ cleavage, but for the coumarin tagged peptides, the local minimum is a stabilized radical on the distributed $\pi$ system, which prevents rapid cleavages. As this peptide then slowly explores the conformational space available, the radical interacts with the side chains enabling further odd-electron rearrangements. In this model, secondary backbone cleavages, while possible, are prevented sterically.

It is interesting to note that the observed loss of backbone fragmentation with the addition of $\mathrm{sp}^{2}$ hybridized "radical trap" moieties is not dissimilar to the loss of fragmentation near the heme group in cytochrome $c$ [18]. In the latter case, it is unclear if the heme is acting as a radical trap or if the iron is changing oxidation state, but the two observations may be related.

\section{Conclusions}

The use of unlabelled, singly-labeled, and doublylabeled Substance P provided an opportunity to study the mechanisms involved in fragment formation under ECD conditions. The addition of the tags served to trap the radical and resulted in decreased abundance of backbone cleavages. It also increased the abundance of side-chain cleavages, which were shown to be derived from a long-lived radical intermediate with the same $\mathrm{m} / \mathrm{z}$ as the charge reduced molecular ion. The enhanced presence of side-chain cleavages in tagged peptides indicated that the addition of the coumarin tags not only prevents backbone cleavages, but redirects the radical to the side chains, presumably by changing the gas-phase conformation of the peptide. This conformational change is also supported by the observation of a previously unreported loss of a phenyl radical, $\mathrm{C}_{6} \mathrm{H}_{5}$, which is speculated to be formed from a conformation in which the coumarin $\mathrm{sp}^{2}$ system interacts with the two phenylalanine side chains via $\pi$-stacking in the gas phase. The implications of these results are intriguing. They may be useful in deducing the mechanism of cleavages in naturally occurring peptides, and could better explain under what conditions and at which locations peptides are likely to be cleaved.

\section{Acknowledgments}

The authors thank Dr. Cheng Zhao, Vera B. Ivleva, Parminder Kaur, Konstantine Aizikov, Raman Mathur, and Professor Catherine E. Costello for experimental assistance and helpful discussions, and NIH/NCRR P41RR10888, NIH/NCRR N01HV28178, MDS Sciex, and the American Chemical Society Petroleum Research Fund for financial support.

\section{References}

1. Zubarev, R. A.; Kelleher, N. L.; McLafferty, F. W. Electron Capture Dissociation of Multiply Charged Protein Cations-A Nonergodic Process. J. Am. Chem. Soc. 1998, 120, 3265-3266.

2. Zubarev, R. A. Reactions of Polypeptide Ions with Electrons in the Gas Phase. Mass Spectrom. Rev. 2003, 22, 57-77.

3. Zubarev, R. A.; Kruger, N. A.; Fridriksson, E. K.; Lewis, M. A.; Horn, D. M.; Carpenter, B. K.; McLafferty, F. W. Electron Capture Dissociation of Gaseous Multiply-Charged Proteins is Favored at Disulfide Bonds and Other Sites of High Hydrogen Atom Affinity. J. Am. Chem. Soc. $1999,121,2857-2862$

4. Kelleher, N. L.; Zubarev, R. A.; Bush, K.; Furie, B.; Furie, B. C.; McLafferty, F. W.; Walsh, C. T. Localization of Labile Post-translational Modifications by Electron Capture Dissociation: The Case of $\gamma$-Carboxyglutamic Acid. Anal. Chem. 1999, 71, 4250-4253.

5. Stensballe, A.; Jensen, O. N.; Olsen, J. V.; Haselmann, K. F.; Zubarev, R. A. Electron Capture Dissociation of Singly and Multiply Phosphorylated Peptides. Rapid Commun. Mass Spectrom. 2000, 14, 1793-1800.

6. Shi, S. D. H.; Hemling, M. E.; Carr, S. A.; Horn, D. M.; Lindh, I.; McLafferty, F. W. Phosphopeptide/Phosphoprotein Mapping by Electron Capture Dissociation Mass Spectrometry. Anal. Chem. 2001, 73, 19-22.

7. Mirgorodskaya, E.; Roepstorff, P.; Zubarev, R. A. Localization of O-Glycosylation Sites in Peptides by Electron Capture Dissociation in a Fourier Transform Mass Spectrometer. Anal. Chem. 1999, 71, 4431-4436.

8. Hakansson, K.; Cooper, H. J.; Emmett, M. R.; Costello, C. E.; Marshall, A. G.; Nilsson, C. L. Electron Capture Dissociation and Infrared Multiphoton Dissociation MS/MS of an N-Glycosylated Tryptic Peptide to Yield Complementary Sequence Information. Anal. Chem. 2001, 73, 4530-4536

9. Patriksson, A.; Adams, C.; Kjeldsen, F.; Raber, J.; van der Spoel, D.; Zubarev, R. A. Prediction of N-C- $\alpha$ Bond Cleavage Frequencies in Electron Capture Dissociation of Trp-Cage Dications by Force-Field Molecular Dynamics Simulations. Int. J. Mass Spectrom. Ion Processes 2006, 248, 124-135.

10. Oh, H.; Breuker, K.; Sze, S. K.; Ge, Y.; Carpenter, B. K.; McLafferty, F. W. Secondary and Tertiary Structures of Gaseous Protein Ions Characterized by Electron Capture Dissociation Mass Spectrometry and Photofragment Spectroscopy. Proc. Nat. Acad. Sci. U.S.A. 2002, 99, 1586315868.

11. Breuker, K.: Oh, H.; Horn, D. M.; Cerda, B. A. McLafferty, F. W Detailed Unfolding and Folding of Gaseous Ubiquitin Ions Characterized by Electron Capture Dissociation. J. Am. Chem. Soc. 2002, 124, 6407-6420.

12. Leymarie, N.; Costello, C. E.; O'Connor, P. B. Electron Capture Dissociation Initiates a Free Radical Reaction Cascade. J. Am. Chem. Soc. 2003, $125,8949-8958$.

13. Leymarie, N.; Berg, E. A.; McComb, M. E.; O'Connor, P. B.; Grogan, J.; Oppenheim, F. G.; Costello, C. E Tandem Mass Spectrometry for Structural Characterization of Proline-Rich Proteins: Application to Salivary PRP-3. Anal. Chem. 2002, 74, 4124-4132.

14. Syrstad, E. A.; Turecek, F. Toward a General Mechanism of Electron Capture Dissociation. J. Am. Soc. Mass Spectrom. 2005, 16, 208-224. 
15. Yao, C. X.; Turecek, F. Hypervalent Ammonium Radicals. Competitive $\mathrm{N}-\mathrm{C}$ and $\mathrm{N}-\mathrm{H}$ Bond Dissociations in Methyl Ammonium and Ethyl Ammonium. Phys. Chem. Chem. Phys. 2005, 7, 912-920.

16. Syrstad, E. A.; Stephens, D. D.; Turecek, F. Hydrogen Atom Adducts to the Amide Bond. Generation and Energetics of Amide Radicals in the Gas Phase. J. Phys. Chem. A 2003, 107, 115-126.

17. O'Connor, P. B.; Lin, C.; Cournoyer, J. J.; Pittman, J. L.; Belyayev, M.; Budnik, B A. Long-Lived Electron Capture Dissociation Product Ions Experience Radical Migration via Hydrogen Abstraction. J. Am. Soc. Mass Spectrom. 2006, 17, 576-585.

18. Horn, D. M.; Breuker, K.; Frank, A. J.; McLafferty, F. W. Kinetic Intermediates in the Folding of Gaseous Protein Ions Characterized by Electron Capture Dissociation Mass Spectrometry. J. Am. Chem. Soc. 2001, 123, 9792-9799.

19. Syrstad, E. A.; Vivekananda, S.; Turecek, F. Direct Observation of a Hydrogen Atom Adduct to C-5 in Uracil. A Neutralization-Reionization Mass Spectrometric and ab initio Study. J. Phys. Chem. A 2001, 105, $8339-8351$.

20. Lin, C.; Cournoyer, J. J.; O'Connor, P. B. Use of a Double Resonance Electron Capture Dissociation Experiment to Probe Fragment Intermediate Lifetimes. J. Am. Soc. Mass Spectrom. 2006, in press.

21. Pashkova, A.; Moskovets, E.; Karger, B. L. Coumarin Tags for Improved Analysis of Peptides by MALDI-TOF MS and MS/MS. 1. Enhancement in MALDI MS Signal Intensities. Anal. Chem. 2004, 76, 4550-4557.
22. O'Connor, P. B.; Pittman, J. L.; Thomson, B. A.; Budnik, B. A.; Cournoyer, J. C.; Jebanathirajah, J.; Lin, C.; Moyer, S A New Hybrid Electrospray Fourier Transform Mass Spectrometer: Design and Performance Characteristics. Rapid Commun. Mass Spectrom. 2006, 20, 259-266.

23. Jebanathirajah, J. A.; Pittman, J. L.; Thomson, B. A.; Budnik, B. A.; Kaur, P.; Rape, M.; Kirschner, M.; Costello, C. E.; O'Connor, P. B. Characterization of a New qQq-FTICR Mass Spectrometer for Post-translational Modification Analysis and Top-Down Tandem Mass Spectrometry of Whole Proteins. J. Am. Soc. Mass Spectrom. 2005, 16, 1985-1999.

24. Cooper, H. J.; Hudgins, R. R.; Hakansson, K.; Marshall, A. G. Characterization of Amino Acid Side-Chain Losses in Electron Capture Dissociation. J. Am. Soc. Mass Spectrom. 2002, 13, 241-249.

25. Kjeldsen, F.; Haselmann, K. F.; Budnik, B. A.; Jensen, F.; Zubarev, R. A. Dissociative Capture of Hot (3-13 eV) Electrons by Polypeptide Polycations: An Efficient Process Accompanied by Secondary Fragmentation. Chem. Phys. Lett. 2002, 356, 201-206.

26. Haselmann, K. F.; Budnik, B. A.; Kjeldsen, F.; Polfer, N. C.; Zubarev, R. A. Can the (M--X) Region in Electron Capture Dissociation Provide Reliable Information on the Amino Acid Composition of Polypeptides? Eur. J. Mass Spectrom. 2002, 8, 461-469.

27. Shaffer, S. A.; Sadilek, M.; Turecek, F. Hypervalent ammonium radicals. Effects of Alkyl Groups and Aromatic Substituents. J. Org. Chem. 1996, $61,5234-5245$. 